\title{
Development of Hydrocarbon Resources on Soft Computing and Data Mining Technologies Base
}

\section{Hasanov RA, Gardashova LA, Kazimov MI, Ramazanov FA and Hasanov}

$\mathrm{AR}^{*}$

Azerbaijan State Oil and Industry University, Azerbaijan

*Corresponding author: Hasanov AR, Azerbaijan State Oil and Industry University, Azerbaijan, Tel: (+99450) 257-24-01; Email: ramizhasanov52@hotmail.com

\section{Research article}

Volume 3 Issue 2

Received Date: May 27, 2019

Published Date: June 26, 2019

DOI: $10.23880 /$ ppej-16000192

\section{Abstract}

Considering of factors characterized by indefiniteness in the statistic methods based development of complex technological processes as well drilling and environment is one of the important requirements. There is need in intellectual analysis of data to eliminate shortage in indefiniteness condition. With these purpose classic methods complex-Fuzzy technology and Data mining technology have been used.

Keywords: Oil well drilling; Statistic methods decision making; Well drilling control; Fuzzy theories; Probability theories; Data mining technology; Intellectual analysis; Fuzzy-C-means algorithm; Clusterization task; Choice of alternatives; Interpretation; Cluster centers; Neuron nets

\section{Methods}

Though there are big resources of mathematical conceptions, methods and theories on decision making in the economy, geologico-technological processes control spheres and other corresponding spheres last decades, complex mathematical theories don't make it possible to solve all existing problems. The reason of it is the intolerableness of mathematical theories to inaccuracy and incompleteness of reality.

A number of works have been devoted to the existing fuzzy logics and probability theories in scientific literature and they are used to consider indefiniteness of medium and information on decision-making in complex technological spheres. There are a number of works in scientific literature concerning development of complex technological process as drilling of wells on the basis of statistic methods [1]. But solution of such task requires considering of factors and medium characterized by indefiniteness. In [2-4] with the purpose of decision making the development of the control system on the well drilling process in indefiniteness condition has been considered.

In geologico-technological researches geological condition, receiving and description of information, prognozing of drilling indices, evaluation of drilling efficiency, information structure used in setting up of dependences of drilling regime parameters are characterized by various types of indefiniteness. Information considered in these spheres consists of mixture of fuzzy and probable indefiniteness. Processing methods of such information has been considered in modern scientific literature. Various scientific approaches are being worked out in this sphere. 


\section{Petroleum \& Petrochemical Engineering Journal}

Geological, technico-technological and ecological information in the process of oil spheres development characterizes the condition expressed by indefiniteness. During the collection and processing the information all attempts made for obtaining complete information are directed to precise information on a whole process, and it requires precise processing of indefinite, fuzzy information. Strong interpretation ability of fuzzy logics, tolerance to indefiniteness make it possible to get complete information.

\section{Results}

Analysis of oil-spheres using statistic methods depending on the information processing brings to various results, and it sometimes conforms partly with the experts' studies in this sphere, sometimes it brings to the opposite results. For eliminating this shortage there is need for intellectual analysis of data. There is a complex of classic methods directed to the increase of oil production coefficient from the layers in the development of oil spheres and currently it is being applied. Dependence of the analyzed data on each other and imprecise analysis of a number of factors influencing the results and limited information due to the shortage of human's calculation ability, form difficulties in making right decision using classic decision making methods.

Comparative analysis of the results obtained by classic methods and intellectual analysis of data have been considered in the article. The suggested approach is based on fuzzy logics and Data mining technology.

\section{Task set up: Investigated Oil Spheres Consist of 24 Objects}

These spheres have been analyzed in scientific literature by statistic methods on the basis of factors influencing oil production within various geological conditions (complexity of geological structure, depth of the deposit, reservoir pressure, length of horizontal area, used equipment and etc.) [4] and object have been divided into 3 groups from exploitation view point, and the most prior object has been determined by classical mathematical logics using oil production coefficients. And as it was mentioned above it doesn't always bring to the real results. Our goal is to determine exactness of the results obtained by classical method using fuzzy technology and Data mining technologies. The results obtained by classical methods are shown in table 1. FussyC-means algorithm will be used to carry out intellectual analysis of the data.
In Fuzzy C- means method used for clustering distance between $X_{i}, i=\overline{1, n}$, objects and $c_{j}, j=\overline{1, n}$ is measured by Euclid norm; $D^{2}=\left\|x_{i}-c_{j}\right\|^{2}$. Other norms are also used in cluster analysis.

Using Euclid distance fuzzy clustering task consists of cluster coordinates providing the following criterion with minimum value and matrix of

$$
J_{m}=\sum_{i=1}^{n} \sum_{j=1}^{n} u_{i j}\left\|x_{i}-c_{j}\right\|^{2}, \quad 1 \leq m<\infty \text { (1) }
$$

here, $c_{j}, j=\overline{1, n}-j-$ cluster center, $\mathrm{m}$ is exponential weight $(m>1), u_{i j}$ belonging function of $x$ on $j-$ cluster, $\|*\|$ is norm reflecting correspondence of cluster centers.

$$
u_{i j}=\frac{1}{\sum_{k=1}^{n}\left(\frac{\left\|x_{i}-c_{j}\right\|}{\left\|x_{i}-c_{k}\right\|}\right)^{\frac{2}{m-1}}}, \quad c_{j}=\frac{\sum_{i}^{n} u_{i j}^{m} x_{i}}{\sum_{i=1}^{n} u_{i j}^{m}}
$$

Value of exponential weight is determined till carrying out clustering and it influences belonging level matrix. More is $\mathrm{M}$, more mixed is the cluster division, when $m \rightarrow \infty$, $M=\left[\frac{1}{c}\right]$. Determination of M value is the task still being researched. In this method when iteration is $\max _{i j}\left\{\left|u_{i j}^{(k+1)}-u_{i j}^{k}\right|\right\}<\varepsilon$ it ends, here $\mathrm{k}$ is iteration steps, $\varepsilon$ is the value varying between 0 and 1 influencing value of purpose function in algorithm iterations. This procedure is collected to local $J_{m}$ minimum.

Fuzzy C-means algorithm consists of the following steps:

1. Initial evaluation of $U=\left[u_{i j}\right], U^{(0)}$ matrix

2. Calculation of k $C^{(k)}=\left[c_{j}\right]$ vectors centers of $U^{(k)}$ matrix in k step.

3. Renovation of values of $U^{(k)}, U^{(k+1)}$ matrix elements 


\section{Petroleum \& Petrochemical Engineering Journal}

$$
c_{j}=\frac{\sum_{i}^{n} u_{i j}^{m} x_{i}}{\sum_{i=1}^{n} u_{i j}^{m}} \quad u_{i j}=\frac{1}{\sum_{k=1}^{n}\left(\frac{\left.\left\|x_{i}-c_{j}\right\|\right)^{\frac{2}{m-1}}}{\left\|x_{i}-c_{k}\right\|}\right)^{n}}
$$

4. $\left\|U^{(k+1)}-U^{(k)}\right\|<\varepsilon$, then process ends, otherwise it should be returned to 2 step.

Fuzzy clustering makes it possible to place data in several clasters. Thus, application of fuzzy clustering gives not only one, but some possible variants, and it widens the choice of alternatives in decision making.

Let's denote cluster centers found as a result of clustering by $v_{1}, v_{2}, \ldots v_{c} \cdot \sum_{k=1}^{n} \mu_{\gamma_{k}}\left(\gamma_{i}\right)=1, i=1, \ldots, c$ expresses the condition that cluster set of fuzzy objects should be closed by fuzzy coating:

For simplicity let's consider that cluster centers are given by two $x$ and $y$ parameters. At this time the main task is the defining of rules expressing the relation between $x$ and $y$. Cluster centers $v_{i}(i=1, \ldots, c)$ are used in writing of fuzzy rule.
If $x=x_{i}$, then $y=y_{i}$, here fuzzy terms are interpreted so: $x_{i}$ is approximate $x_{i}$ and $y_{i}$ is approximate $y_{i}$ : belonging functions of fuzzy terms are expressed by Gause curve:

$$
x_{i}=\exp \left(-\frac{1}{2}\left(\frac{x-x_{i}}{\beta}\right)^{2}\right), \quad y_{i}=\exp \left(-\frac{1}{2}\left(\frac{y-y_{i}}{\beta}\right)^{2}\right)
$$

here $\beta-$ is the parameter of clustering algorithm.

Computer simulation: Let's consider creation of 3, 5 and 7 group using existing information and choice of priority object. Initial data of the task is as in table 1:

\section{Initial Data}

1. $\quad$ Name of oil deposit; 2. Exploitation object, 3. The depth of geological deposit; 4 . The area having oil recovery ability, 5. Effective thickness, 6. Porosity, 7. Permeability, 8. Density, 9. Extracted oil, 10. Oil production (current and final) Goal: creation of groups 3, 5 and 7 , and choice of priority object.

\begin{tabular}{|c|c|c|c|c|c|c|c|c|c|c|c|}
\hline \multirow[t]{2}{*}{ № } & \multirow[t]{2}{*}{ Deposit } & \multirow{2}{*}{$\begin{array}{c}\text { Exploita } \\
\text { tion } \\
\text { Object }\end{array}$} & \multirow{2}{*}{\begin{tabular}{|c|} 
Depth \\
of \\
Bedding
\end{tabular}} & \multirow{2}{*}{$\begin{array}{c}\text { pil Bearin } \\
\text { Area, 10 } \\
\mathbf{M}^{2}\end{array}$} & \multirow{2}{*}{$\begin{array}{c}\text { Effective } \\
\text { Thickness } \\
\text { M }\end{array}$} & \multirow{2}{*}{$\begin{array}{c}\text { Porosity } \\
\%\end{array}$} & \multirow{2}{*}{$\begin{array}{c}\text { Reducibility } \\
10^{-3} \mathrm{~mm}\end{array}$} & \multirow{2}{*}{$\begin{array}{c}\text { Oil } \\
\text { Density } \\
\mathrm{Kq} / \mathrm{M}^{3}\end{array}$} & \multirow{2}{*}{\begin{tabular}{|c} 
Total \\
Production \\
Th/T
\end{tabular}} & \multicolumn{2}{|c|}{$\begin{array}{c}\text { Oil Recovery } \\
\text { Coefficient }\end{array}$} \\
\hline & & & & & & & & & & \begin{tabular}{|l|} 
Current \\
\end{tabular} & Final \\
\hline \multicolumn{12}{|c|}{ I group objects } \\
\hline 1 & Umbakl & Maykop & 1380 & 856 & 23,8 & 20 & 46 & 895 & 943 & 0,04 & 0,2 \\
\hline 2 & Neftchala & $\mathrm{V}$ & 125 & 442 & 27,1 & 24 & 182 & 865 & 617 & 0,05 & 0,18 \\
\hline 3 & Zeyli & Miosen & 1750 & 488 & 75 & 18 & 38 & 845 & 911 & 0,08 & 0,21 \\
\hline 4 & Umbakl & Çokrak & 600 & 206 & 28,7 & 20 & 122 & 950 & 408 & 0,05 & 0,10 \\
\hline 5 & Neftchala & IV & 985 & 349 & 19,5 & 24 & 160 & 922 & 299 & 0,04 & 0,14 \\
\hline 6 & Kalamaddin & IV & 1450 & 12 & 57,7 & 22 & 20 & 907 & 461 & 0,07 & 0,36 \\
\hline 7 & Neftchala & VII & 1487 & 428 & 16,2 & 25 & 57 & 8850 & 386 & 0,06 & 0,2 \\
\hline 8 & Neftchala & III & 807 & 234 & 15,5 & 24 & 263 & 891 & 267 & 0,05 & 0,19 \\
\hline 9 & Kalamaddin & III & 1350 & 90 & 42,4 & 22 & 18 & 910 & 135 & 0,03 & 0,35 \\
\hline \multicolumn{12}{|c|}{ II group objects } \\
\hline 1 & Karabakhli & VII & 3610 & 516 & 23,7 & 21 & 68 & 892 & 639 & 0,05 & 0,2 \\
\hline 2 & Old Kala & $\mathrm{KC}$ & 2150 & 441 & 14,5 & 21 & 123 & 889 & 552 & 0,07 & 0,30 \\
\hline 3 & Karabakhli & $\mathrm{V}$ & 3350 & 494 & 17,3 & 22 & 30 & 899 & 613 & 0,07 & 0,19 \\
\hline 4 & Zeyva & V & 2550 & 450 & 20,4 & 24 & 27 & 895 & 414 & 0,05 & 0,09 \\
\hline 5 & Karabakhli & III & 3150 & 480 & 14,3 & 22 & 76 & 896 & 445 & 0,06 & 0,19 \\
\hline 6 & Karadagh & Diatom & 2750 & 744 & 28,3 & 16 & 56 & 910 & 44 & 0,01 & 0,1 \\
\hline 7 & Jafarli & Eosen & 3920 & 637 & 50,6 & 4 & 36 & 879 & 248 & 0,04 & 0,1 \\
\hline 8 & Zeyva & VI & 2670 & 251 & 19,6 & 22 & 30 & 879 & 168 & 0,03 & 0,07 \\
\hline 9 & C. Kyursanqa & VIII & 3935 & 213 & 17,5 & 21 & 38 & 895 & 104 & 0,02 & 0,16 \\
\hline 10 & Zeyva & VIII & 2910 & 166 & 24 & 24 & 92 & 873 & 218 & 0,05 & 0,08 \\
\hline
\end{tabular}

Initial data of the task are given in Table 1. 


\section{Petroleum \& Petrochemical Engineering Journal}

\begin{tabular}{|c|c|c|c|c|c|c|c|c|c|c|c|}
\hline 11 & Karabakhli & IV & 3250 & 194 & 11,2 & 23 & 19 & 901 & 232 & 0,05 & 0,21 \\
\hline \multicolumn{12}{|c|}{ III group objects } \\
\hline 1 & $\begin{array}{l}\text { Kyursanga } \\
\text { south }\end{array}$ & XIII & 4765 & 429 & 20,3 & 20 & 27 & 895 & 32 & 0,003 & 0,15 \\
\hline 2 & Neftchala & XIII & 4100 & 474 & 13,2 & 18 & 50 & 889 & 66 & 0,01 & 0,2 \\
\hline 3 & Kalamaddin & Eosen & 4050 & 544 & 48,5 & 28 & 4 & 882 & 195 & 0,04 & 0,12 \\
\hline 4 & $\begin{array}{l}\text { Kyursanga } \\
\text { south }\end{array}$ & XIV & 5000 & 413 & 12,4 & 22 & 27 & 889 & 2 & 0,001 & 0,2 \\
\hline
\end{tabular}

Table 1: Initial information base.

Data used for obtaining fuzzy clusters are as follows. Exponent is $\mathrm{m}=3$; improvement constant $=0.000$ 001; number of iterations is 10000; number of clusters is 3,5 , 7,9 .

In the case when number of clusters is 3 centers of the clusters obtained on the basis of intellectual analysis of data and values of belonging functions are as follows.

Iteration values according to calculation:

Iteration count $=1$, obj. $\mathrm{fcn}=16903744.071569$

Iteration count $=2$, obj. $\mathrm{fcn}=12928316.867672$

Iteration count $=3$, obj. $\mathrm{fcn}=9610738.100440$

Iteration count $=4$, obj. $\mathrm{fcn}=6611479.254957$

Iteration count $=5$, obj. $\mathrm{fcn}=5458026.748869$

Iteration count $=6$, obj. $\mathrm{fcn}=4953940.811972$

Iteration count $=7$, obj. $\mathrm{fcn}=4724454.476005$

Iteration count $=8$, obj. $\mathrm{fcn}=4631882.469071$

Iteration count $=9$, obj. $\mathrm{fcn}=4598449.067768$

Iteration count $=10$, obj. $\mathrm{fcn}=4587058.272361$

Iteration count $=11, \mathrm{obj}$. $\mathrm{fcn}=4583279.774332$

Iteration count $=12$, obj. $\mathrm{fcn}=4582041.535835$

Iteration count $=13$, obj. $\mathrm{fcn}=4581638.077309$

Iteration count $=14, \mathrm{obj}$. $\mathrm{fcn}=4581506.988790$

Iteration count $=15$, obj. $\mathrm{fcn}=4581464.458463$

Iteration count $=16$, obj. $\mathrm{fcn}=4581450.670592$

Iteration count $=17, \mathrm{obj}$. $\mathrm{fcn}=4581446.202593$

Iteration count $=18$, obj. $\mathrm{fcn}=4581444.755061$

Iteration count $=19$, obj. $\mathrm{fcn}=4581444.286155$

Iteration count $=20$, obj. $\mathrm{fcn}=4581444.134271$

Iteration count $=21$, obj. $\mathrm{fcn}=4581444.085075$

Iteration count $=22$, obj. $\mathrm{fcn}=4581444.069142$

Iteration count $=23$, obj. $\mathrm{fcn}=4581444.063981$

Iteration count $=24$, obj. $\mathrm{fcn}=4581444.062309$

Iteration count $=25$, obj. $\mathrm{fcn}=4581444.061768$

Iteration count $=26$, obj. $\mathrm{fcn}=4581444.061593$

Iteration count $=27, \mathrm{obj}$. $\mathrm{fcn}=4581444.061536$

Iteration count $=28$, obj. $\mathrm{fcn}=4581444.061518$

Iteration count $=29$, obj. $\mathrm{fcn}=4581444.061512$

Cluster centers

Center $=1.0 \mathrm{e}+003^{*}$ $\begin{array}{llllllll}2.8991 & 0.3967 & 0.0200 & 4.2272 & 0.4581 & 0.0270 & 1.2058\end{array}$

0.33000 .0315

$\begin{array}{lllllll}0.0218 & 0.0536 & 0.8916 & 0.0192 & 0.0320 & 0.8884 & 0.0224\end{array}$

0.10790 .8984

$\begin{array}{lllllll}0.3267 & 0.0000 & 0.0001 & 0.1379 & 0.0000 & 0.0002 & 0.4605\end{array}$ 0.00010 .0002

Values of belonging function:

$\begin{array}{lll}0.1502 & 0.0489 & 0.8009\end{array}$

$\begin{array}{lll}0.0151 & 0.0050 & 0.9799\end{array}$

$\begin{array}{lll}0.2279 & 0.0567 & 0.7155\end{array}$

$\begin{array}{lll}0.0660 & 0.0265 & 0.9076\end{array}$

$\begin{array}{lll}0.0208 & 0.0072 & 0.9720\end{array}$

$\begin{array}{lll}0.0681 & 0.0193 & 0.9127\end{array}$

$\begin{array}{lll}0.0458 & 0.0121 & 0.9421\end{array}$

$\begin{array}{lll}0.0483 & 0.0182 & 0.9336\end{array}$

$\begin{array}{lll}0.0692 & 0.0208 & 0.9099\end{array}$

$\begin{array}{lll}0.4819 & 0.4672 & 0.0509\end{array}$

$\begin{array}{lll}0.5508 & 0.0758 & 0.3734\end{array}$

$\begin{array}{lll}0.7354 & 0.2179 & 0.0467\end{array}$

$\begin{array}{lll}0.8938 & 0.0412 & 0.0650\end{array}$

$\begin{array}{lll}0.9180 & 0.0616 & 0.0204\end{array}$

$\begin{array}{lll}0.8475 & 0.0832 & 0.0692\end{array}$

$\begin{array}{lll}0.1099 & 0.8739 & 0.0162\end{array}$

$\begin{array}{lll}0.9218 & 0.0372 & 0.0410\end{array}$

$\begin{array}{lll}0.1107 & 0.8724 & 0.0169\end{array}$

$\begin{array}{lll}0.9443 & 0.0346 & 0.0211\end{array}$

$\begin{array}{lll}0.8266 & 0.1395 & 0.0339\end{array}$

$\begin{array}{lll}0.0762 & 0.9026 & 0.0211\end{array}$

$\begin{array}{lll}0.0143 & 0.9831 & 0.0025\end{array}$

$\begin{array}{lll}0.0306 & 0.9643 & 0.0051\end{array}$

$\begin{array}{lll}0.1160 & 0.8482 & 0.0359\end{array}$

As a result, obtained groups distinguish significantly from the results obtained by classic method. Here, priority object is the object, which has obtained the biggest value of belonging function.

The obtained result is the object providing entrance to the group not only on one or some factors, but with total highest belonging level of all factors. The obtained fuzzy clusters are given in Figure 1. 

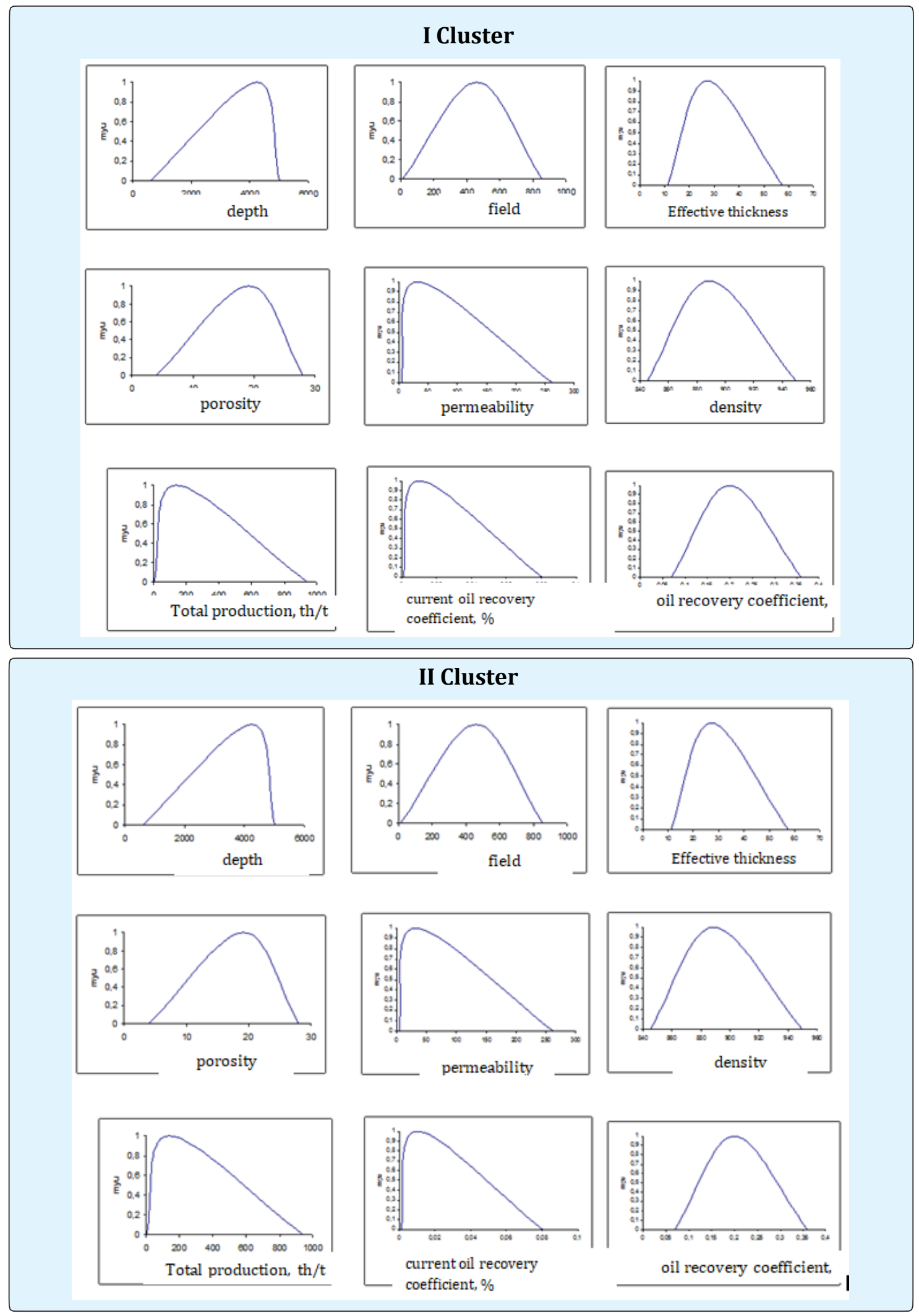


\section{Petroleum \& Petrochemical Engineering Journal}
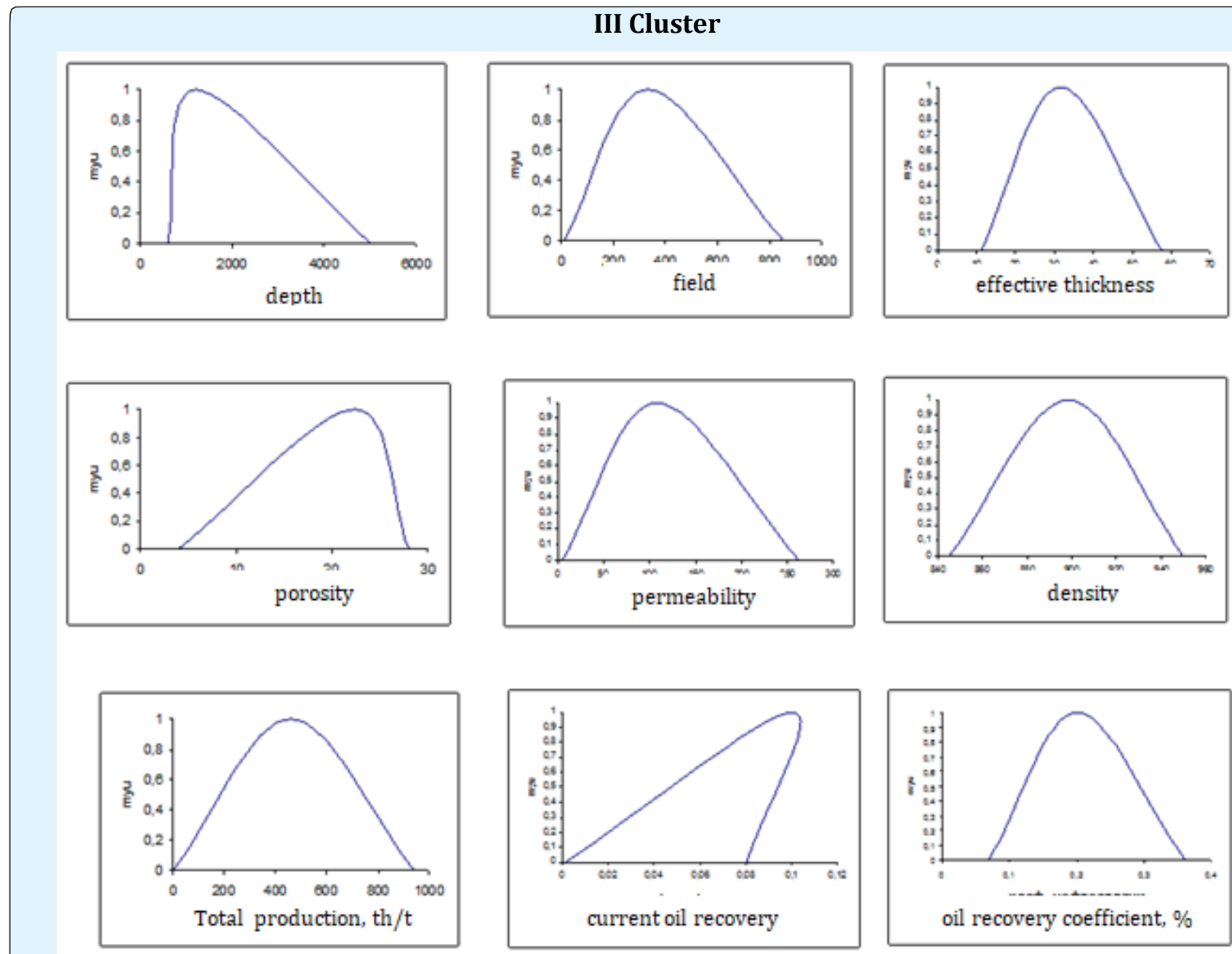

Figure 1: Description of fuzzy clusters.

The obtained groups are given in Table 2 .

\begin{tabular}{|c|c|c|c|c|c|c|c|c|c|c|c|}
\hline \multirow{2}{*}{ № } & \multirow{2}{*}{ Deposit } & \multirow{2}{*}{$\begin{array}{c}\text { Exploitation } \\
\text { Object }\end{array}$} & \multirow{2}{*}{$\begin{array}{c}\text { Depth } \\
\text { of } \\
\text { Bedding }\end{array}$} & \multirow{2}{*}{\begin{tabular}{|c|} 
Oil \\
Bearing \\
Area, 104 \\
$\mathrm{M}^{2}$ \\
\end{tabular}} & \multirow{2}{*}{$\begin{array}{c}\text { Effective } \\
\text { Thickness, } \\
\text { M }\end{array}$} & \multirow{2}{*}{$\begin{array}{c}\text { Porosity } \\
\%\end{array}$} & \multirow{2}{*}{$\begin{array}{c}\text { Permeability, } \\
10^{-3} \mathrm{~mm}\end{array}$} & \multirow{2}{*}{ Oil } & \multirow{2}{*}{$\begin{array}{c}\text { Total } \\
\text { Production, } \\
\text { Th/T }\end{array}$} & \multicolumn{2}{|c|}{$\begin{array}{c}\text { Oil Recovery } \\
\text { Coefficient }\end{array}$} \\
\hline & & & & & & & & & & Curren & Final \\
\hline \multicolumn{12}{|c|}{ I group objects } \\
\hline 1 & Karabakhli & $\mathrm{V}$ & 3350 & 494 & 17,3 & 22 & 30 & 899 & 613 & 0,07 & 0,19 \\
\hline 2 & Zeyba & $\mathrm{V}$ & 2550 & 450 & 20,4 & 24 & 27 & 895 & 414 & 0,05 & 0,09 \\
\hline 3 & Karabakhli & III & 3150 & $4 \$ 6$ & 14,3 & 22 & 76 & 896 & 445 & 0,06 & 0,19 \\
\hline 4 & Karadakh & Diatom & 2750 & 744 & 28,3 & 16 & 5656 & 910 & 44 & 0,01 & 6,1 \\
\hline 5 & Zeyba & VI & 2476 & 251 & 19,6 & 22 & 56 & 879 & 168 & 6,65 & 6,67 \\
\hline 6 & Zeyba & VIII & 2910 & 166 & 24 & 24 & 92 & 873 & 215 & 6,65 & $6,6 \mathrm{~s}$ \\
\hline 7 & Karabakhli & IV & 3250 & 194 & 11,2 & 23 & 19 & 901 & 232 & 0,05 & 0,21 \\
\hline 8 & Karabakhli & VII & 3610 & 516 & 23,7 & 21 & $6 S$ & 892 & 639 & 0,05 & 0,2 \\
\hline
\end{tabular}




\section{Petroleum \& Petrochemical Engineering Journal}

\begin{tabular}{|c|c|c|c|c|c|c|c|c|c|c|c|}
\hline 9 & Old Qala & $\mathrm{KC}$ & 2150 & 441 & 14.5 & 21 & 123 & 889 & 552 & 0.07 & 0.30 \\
\hline \multicolumn{12}{|c|}{ II group objects } \\
\hline 1 & Jafarli & ET & 3920 & 637 & 50,6 & 4 & 36 & 879 & 248 & 0,04 & 0,1 \\
\hline 2 & $\begin{array}{c}\text { North } \\
\text { Kyursanga }\end{array}$ & VIII & 3935 & 213 & 17,5 & 21 & 38 & S95 & 104 & 0,02 & 0,16 \\
\hline 3 & $\begin{array}{c}\text { Kyursanga } \\
\text { south }\end{array}$ & XIII & 4765 & 429 & 20,3 & 20 & 27 & S95 & 32 & 0,003 & 0.15 \\
\hline 4 & Neftchala & XIII & 4100 & 474 & 13,2 & 18 & 50 & SS9 & 66 & 0,01 & 0,2 \\
\hline & Kalamaddin & ET & 4050 & 544 & 48,5 & 28 & 4 & 882 & 195 & 0,04 & 0.12 \\
\hline 6 & $\begin{array}{c}\text { Kyursanga } \\
\text { south }\end{array}$ & XIV & 5666 & 415 & 12,4 & 22 & 27 & 889 & 2 & 6,661 & 6,2 \\
\hline \multicolumn{12}{|c|}{ III group objects } \\
\hline 1 & Umbakl & MA & 1380 & 856 & 23.8 & 20 & 46 & 895 & 943 & 0,04 & 0,2 \\
\hline 2 & Neftchala & $\mathrm{V}$ & 1125 & 442 & 27.1 & 24 & 182 & 865 & 617 & 0.05 & 0.18 \\
\hline 3 & Zeybi & $\mathrm{M}$ & 1750 & 488 & 75 & 18 & 38 & 845 & 911 & 0,08 & 0,21 \\
\hline 4 & Umbaki & Ç & 600 & 206 & 28,7 & 20 & 122 & 950 & 408 & 0.05 & 0.10 \\
\hline 5 & Neftchala & IV & 985 & 349 & 19.5 & 24 & 160 & 922 & 299 & 0.04 & 0.14 \\
\hline \multicolumn{2}{|c|}{6 Kalamaddin } & IV & 1450 & 12 & 57,7 & 22 & 20 & 907 & 461 & 0,07 & 0,36 \\
\hline 7 & Neftchala & VII & 1487 & 428 & 16,2 & 25 & 57 & 850 & 386 & 0,06 & 0,2 \\
\hline 8 & Neftchala & III & 807 & 234 & 15.5 & 24 & 263 & 891 & 267 & 0.05 & 0.19 \\
\hline \multicolumn{2}{|c|}{9 Kalamaddin } & III & 1350 & 90 & 42,4 & 22 & 18 & 910 & 135 & 0,03 & 0,35 \\
\hline
\end{tabular}

Table 2:

When the number of clusters is 5 , cluster centers obtained on the basis of data intellectual analyses and fragment of belonging functions values are given below.

Iteration values according to calculation:

Iteration count $=1$, obj. $\mathrm{fcn}=10342361.076339$

Iteration count $=2$, obj. $\mathrm{fcn}=5287085.833270$

Iteration count $=3$, obj. $\mathrm{fcn}=3278833.976970$

Iteration count $=79$, obj. $\mathrm{fcn}=2144330.957163$

Iteration count $=80$, obj. $\mathrm{fcn}=2144330.957137$

Iteration count $=81$, obj. $\mathrm{fcn}=2144330.957119$

Iteration count $=82$, obj. $\mathrm{fcn}=2144330.957107$

Iteration count $=83$, obj. $\mathrm{fcn}=2144330.957100$

Results obtained on Fuzzy C-means method ( 3 groups on the objects)

\begin{tabular}{|l|l|l|l|l|l|l|l|l|}
\hline 2.6875 & 0.3646 & 0.3646 & 0.0220 & 0.0480 & 0.8874 & 0.2435 & 0.0000 & 0.0001 \\
0.8308 & 0.2731 & 0.2731 & 0.0228 & 0.1854 & 0.9148 & 0.3410 & 0.0000 & 0.0002 \\
1.4228 & 0.1746 & 0.1746 & 0.0228 & 0.0399 & 0.9003 & 0.3533 & 0.0001 & 0.0003 \\
1.6079 & 0.6193 & 0.6193 & 0.0193 & 0.0511 & 0.8684 & 0.8770 & 0.0001 & 0.0002 \\
\hline
\end{tabular}

$\begin{array}{llllllllll}3.3018 & 0.4519 & 0.0170 & 0.0219 & 0.0507 & 0.8968 & 0.4995 & 0.0001 & 0.0002\end{array}$

$\begin{array}{lllllllll}4.8766 & 0.4207 & 0.0164 & 0.0210 & 0.0271 & 0.8920 & 0.0180 & 0.0000 & 0.0002\end{array}$

$\begin{array}{lllllllll}3.9986 & 0.4841 & 0.0332 & 0.0183 & 0.0312 & 0.8858 & 0.1618 & 0.0000 & 0.0001\end{array}$

Values of belonging function (fragment)

$\begin{array}{lllll}0.0139 & 0.0623 & 0.2070 & 0.0257 & 0.6911\end{array}$

$\begin{array}{lllll}0.0058 & 0.0259 & 0.6404 & 0.0105 & 0.3174\end{array}$

$\begin{array}{lllll}0.0061 & 0.0374 & 0.0635 & 0.0123 & 0.8808\end{array}$

$\begin{array}{lllll}0.0066 & 0.0229 & 0.8584 & 0.0109 & 0.1013\end{array}$

0.03010 .01520 .00220 .94910 .0034

0.97710 .00430 .00120 .01590 .0016 


\section{Petroleum \& Petrochemical Engineering Journal}

The groups obtained by using 5 clusters are given in Table 3.

\begin{tabular}{|c|c|c|c|c|c|c|c|c|c|c|c|}
\hline \multirow{2}{*}{ № } & \multirow{2}{*}{ Deposit } & \multirow{2}{*}{$\begin{array}{c}\text { Exploitation } \\
\text { Object }\end{array}$} & \multirow{2}{*}{$\begin{array}{l}\text { Depth of } \\
\text { Bedding }\end{array}$} & \multirow{2}{*}{$\begin{array}{c}\text { Oil } \\
\text { Bearing } \\
\text { Area, } \\
\text { 10 }^{4} \mathbf{M}^{2}\end{array}$} & \multirow{2}{*}{\begin{tabular}{|c|} 
Effective \\
Thickness, \\
M
\end{tabular}} & \multirow{2}{*}{$\begin{array}{c}\text { Porosity, } \\
\%\end{array}$} & \multirow{2}{*}{$\begin{array}{c}\text { Permeability, } \\
10^{-3} \mathrm{~mm}\end{array}$} & \multirow{2}{*}{$\begin{array}{c}\text { Oil } \\
\text { Density }\end{array}$} & \multirow{2}{*}{$\begin{array}{c}\text { Total } \\
\text { Production, } \\
\text { Th/T }\end{array}$} & \multicolumn{2}{|c|}{$\begin{array}{c}\text { Oil Recovery } \\
\text { Coefficient }\end{array}$} \\
\hline & & & & & & & & & & Current & Final \\
\hline \multicolumn{12}{|c|}{ I group objects } \\
\hline 1 & Kyursanga & XIII & 4765 & 429 & 20.3 & 20 & 27 & 895 & 32 & 0.003 & 0.15 \\
\hline 2 & Kyursanga & XIV & 5000 & 413 & 12.4 & 22 & 27 & 889 & 2 & 0.001 & 0.2 \\
\hline \multicolumn{12}{|c|}{ II group objects } \\
\hline 1 & Zeyba & $\mathrm{V}$ & 2550 & 450 & 20.4 & 24 & 27 & 895 & 414 & 0.05 & 0.09 \\
\hline 2 & Karabakhli & III & 3150 & 480 & 14.3 & 22 & 76 & 896 & 445 & 0.06 & 0.19 \\
\hline 3 & Karabakhli & $\mathrm{D}$ & 2750 & 744 & 28.3 & 16 & 56 & 910 & 44 & 0.01 & 0.1 \\
\hline 4 & Zeyba & VI & 2670 & 251 & 19.6 & 22 & 30 & 879 & 168 & 0.03 & 0.07 \\
\hline 5 & Zeyba & VIII & 2910 & 166 & 24 & 24 & 92 & 873 & 218 & 0.05 & $0.0 \mathrm{~S}$ \\
\hline 6 & Karabakhli & IV & 3250 & 194 & 11.2 & 23 & 19 & 901 & 232 & 0.05 & 0.21 \\
\hline \multicolumn{12}{|c|}{ III group objects } \\
\hline 1 & Neftchala & $\mathrm{V}$ & 1125 & 442 & 27.1 & 24 & 182 & 865 & 617 & 0.05 & $0.1 \mathrm{~S}$ \\
\hline 2 & Umbakl & $\mathrm{C}$ & 600 & 206 & 28,7 & 20 & 122 & 950 & $40 \mathrm{~S}$ & 0.05 & 0.10 \\
\hline 3 & Neftchala & IV & 985 & 349 & 19.5 & 24 & 160 & 922 & 299 & 0.04 & 0.14 \\
\hline 4 & Neftchala & III & 807 & 234 & 15.5 & 24 & 263 & 891 & 267 & 0.05 & 0.19 \\
\hline 5 & Kalamaddin & III & 1350 & 90 & 42.4 & 22 & $1 \mathrm{~S}$ & 910 & 135 & 0.03 & 0.35 \\
\hline \multicolumn{12}{|c|}{ IV group of object } \\
\hline 1 & Jafarli & $\bar{E}$ & 3920 & 637 & 50.6 & 4 & 36 & 879 & 248 & 0.04 & 0.1 \\
\hline 2 & $\begin{array}{c}\text { South } \\
\text { Karsanqa }\end{array}$ & VIII & 3935 & 213 & 17.5 & 21 & 38 & 895 & 104 & 0.02 & 0.16 \\
\hline 3 & Neftchala & XIII & 4100 & 474 & 13.2 & 18 & 50 & 889 & 66 & 0.01 & 0.2 \\
\hline 4 & Kalamaddin & $\mathrm{E}$ & 4050 & 544 & 48.5 & 28 & 4 & 882 & 195 & 0.04 & 0.12 \\
\hline 5 & Karabakhli & VII & 3610 & 516 & 23,7 & 21 & 68 & 892 & 639 & 0.05 & 0.2 \\
\hline \multicolumn{12}{|c|}{ V group of object } \\
\hline 1 & Umbakı & $\mathrm{M}$ & 1380 & 856 & 23.8 & 20 & 46 & 895 & 943 & 0.04 & 0.2 \\
\hline 2 & Zeyba & $\mathrm{M}$ & 1750 & 488 & 75 & 18 & 38 & 845 & 911 & 0.08 & 0.21 \\
\hline 3 & Neftchala & VII & 1487 & 428 & 16.2 & 25 & 57 & 8850 & 386 & 0.06 & 0.2 \\
\hline 4 & $\begin{array}{c}\text { Kyursanga } \\
\text { south }\end{array}$ & KS & 2150 & 441 & 14.5 & 21 & 123 & 889 & 552 & 0.07 & 0.30 \\
\hline 5 & Kalamaddin & IV & 1450 & 12 & 57.7 & 22 & 20 & 907 & 461 & 0.07 & 0.36 \\
\hline
\end{tabular}

Table 3: Results obtained by 5 clusters.

The same tests have been carried out in the case when number of clusters is 7 and 9 . The obtained results make it possible to generalize expert's knowledge working in the field of oil wells drilling and to make better decisions.

In the paper, data are used to get knowledge and intellectual analyses have been carried out by fuzzy neuron nets. This approach makes it possible to determine priority of the object entered the group by including expert's any statistic information.
Due to the used Soft computing technology the algorithm is based on fuzzy neuron net. Work principle of fuzzy neuron net is given below [5-8]. For simplicity fuzzy neuron net can be explained on the neuron net having 2 inlets and one outlet Typical rule set for Sugeno fuzzy model having two fuzzy "If...then..." rule is expressed as follows:

If $\left(\begin{array}{ll}x_{1} & A_{1}\end{array}\right)$ and $\left(x_{2} \quad B_{1}\right)$ Then $f_{1}=p_{1} x_{1}+q_{1} x_{2}+r_{1}$ 


\section{Petroleum \& Petrochemical Engineering Journal}

If $\left(\begin{array}{ll}x_{1} & A_{2}\end{array}\right)$ and $\left(x_{2} \quad B_{2}\right)$ Then $f_{2}=p_{2} x_{1}+q_{2} x_{2}+r_{2}$

ANFES (adaptive neuro fuzzy extract system) has 5 layers onward direction structure. The positions of these layers are as follows.

Layer-1: Each $i$ knot taking part in this layer is the adaptive knot the output of which is determined as follows:

$a_{1, i}=\mu_{A_{i}}\left(x_{1}\right), i=1,2$ and $a_{1, i}=\mu_{B_{i \rightarrow \infty}}\left(x_{2}\right), i=3,4$

Layer-2: Each knot in this layer is the constant removing sum of the coming signals and indicated as II. The output of the 2-nd layer can be described as follows:

$$
a_{2, i}=w_{i}=\mu_{A_{i}}\left(x_{1}\right) \cdot \mu_{B_{i}}\left(x_{2}\right), \quad i=1,2
$$

Layer-3: Each knot taking part in the 3-rd layer is the constant knot indicated as $\mathrm{N}$.

Each i knot in this layer calculates the ratio of reality level of i-rule to the sum of reality levels of all rules:

$$
a_{3, i}=\bar{w}_{i}=\frac{w_{i}}{w_{1}+w_{2}}, \quad i=1,2
$$

Layer-4: Each i knot of this layer is the adaptive knot as follows:

$$
a_{4, i}=w_{i} f_{i}=\bar{w}_{i}\left(p_{i} x_{1}+q_{i} x_{2}+r_{i}\right)
$$

Layer-5: In the last fifth layer the only constant knot receiving all coming signals is the knot calculating $\Sigma$ indicated and sum output. The output of this knot is written as follows:

$$
a_{5, i}=\text { sum output }=\sum_{i} \bar{w}_{i} f_{i}=\frac{\sum_{i} \bar{w}_{i} f_{i}}{\sum_{i} \bar{w}_{i}}
$$

Let's consider computer simulation on fuzzy neuron net operating above. Study has been carried out including initial data in the inlet of fuzzy neuron net. Description of the initial data is given in Figure 2.

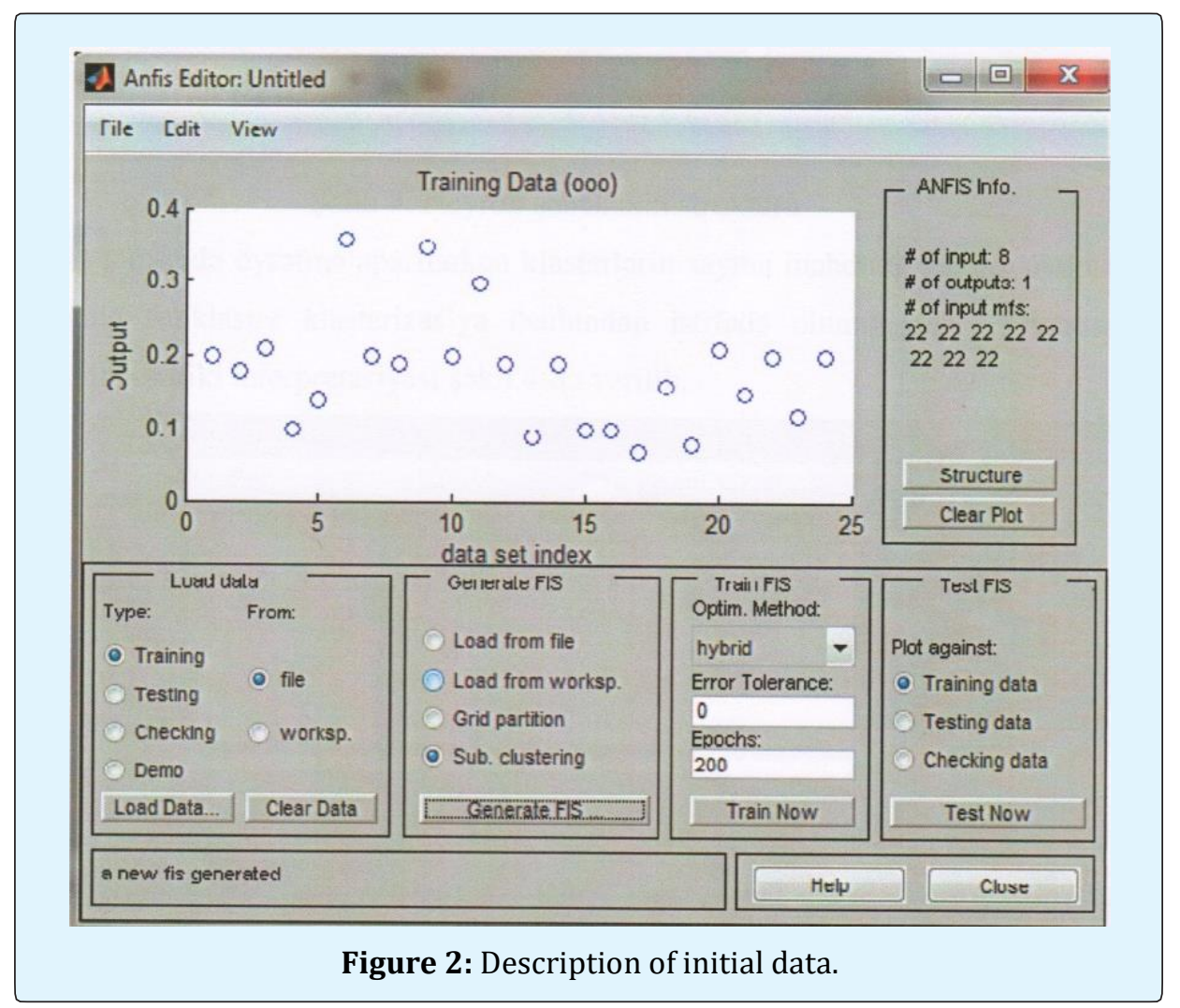




\section{Petroleum \& Petrochemical Engineering Journal}

The structure of the used neuron net is given in Figure 3.

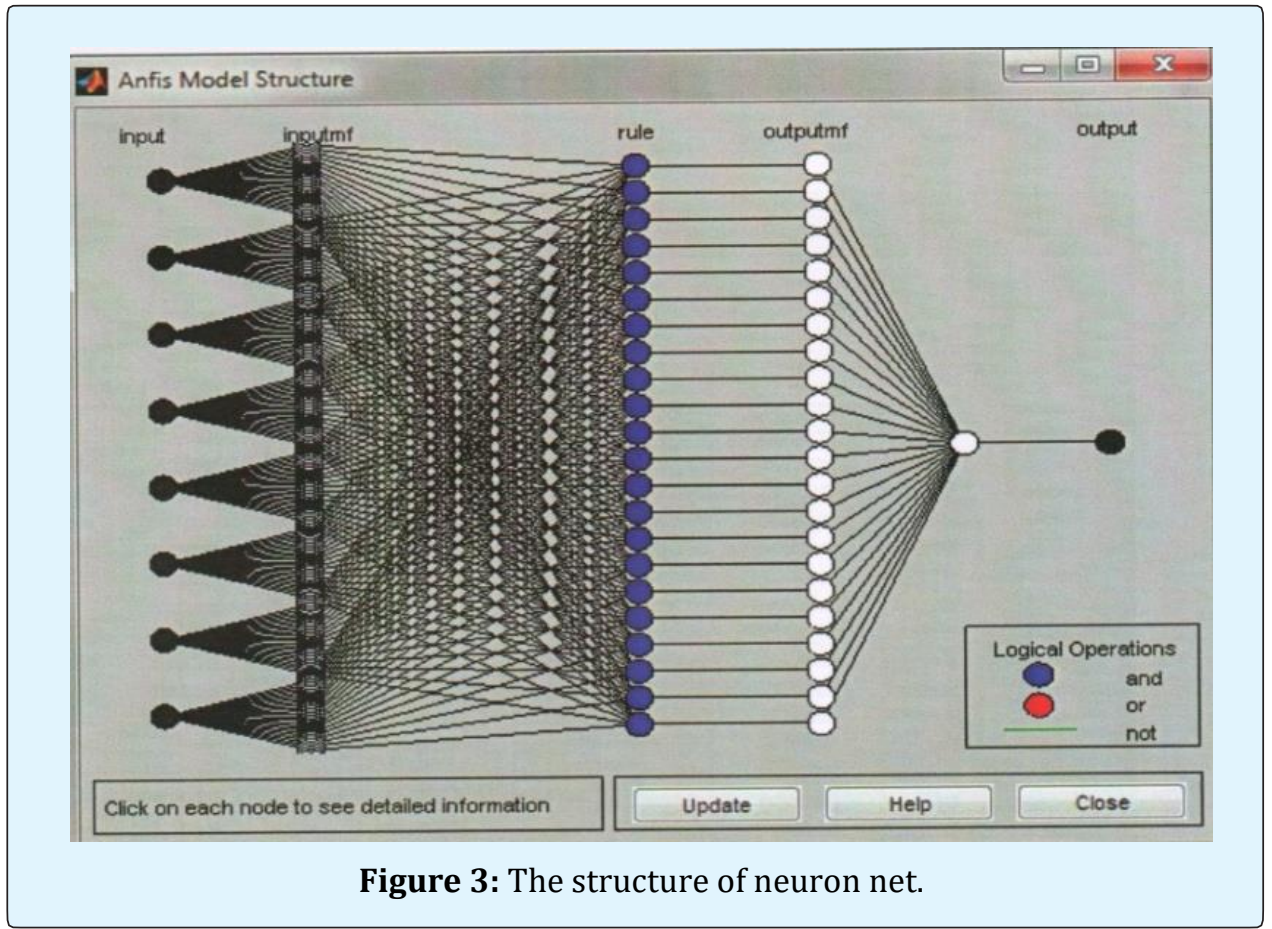

When carrying out study in neuron net sub cluster cluster number. Interpretation graphics of the study clusterisation method has been used not to limit the process is given in Figure 4.

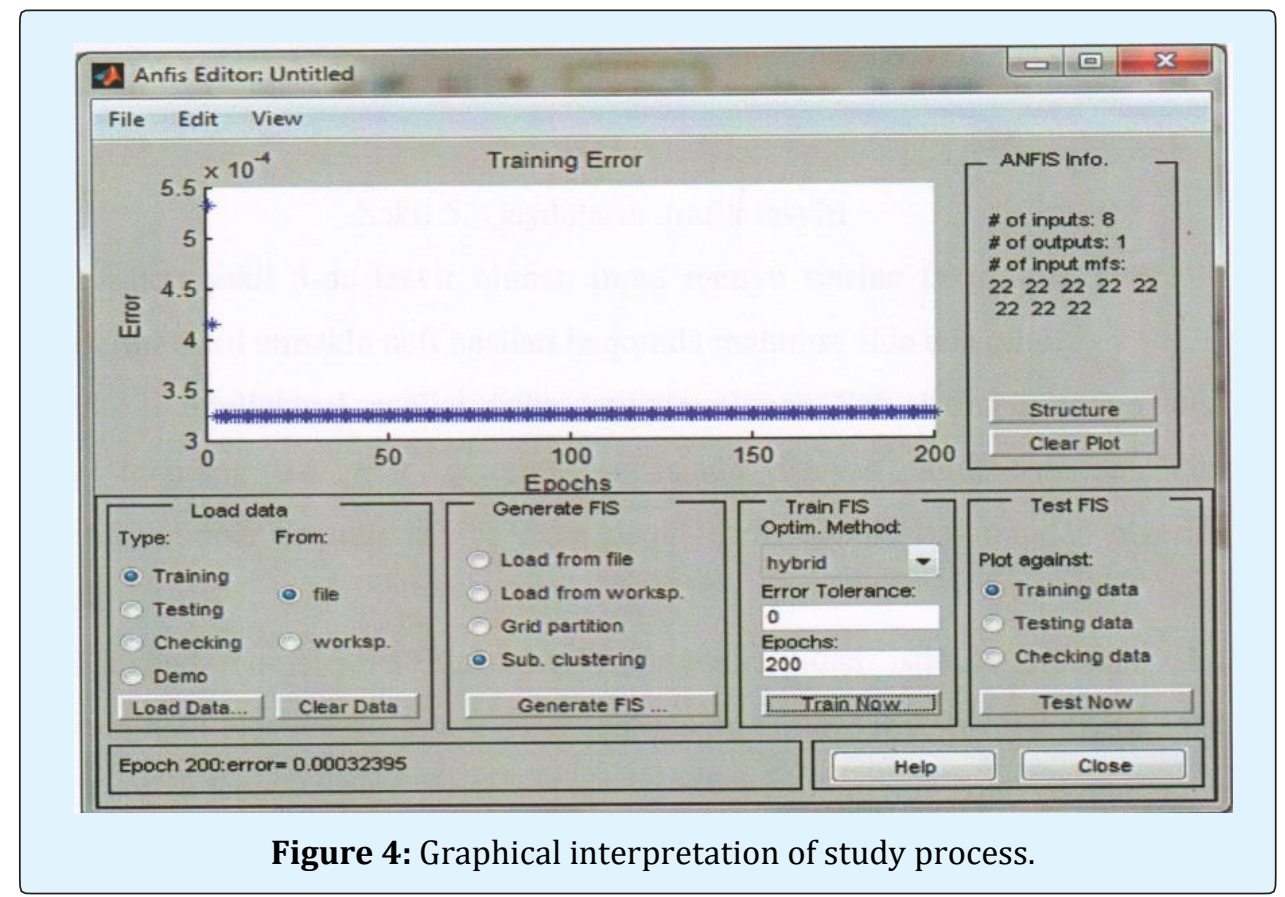




\section{Petroleum \& Petrochemical Engineering Journal}

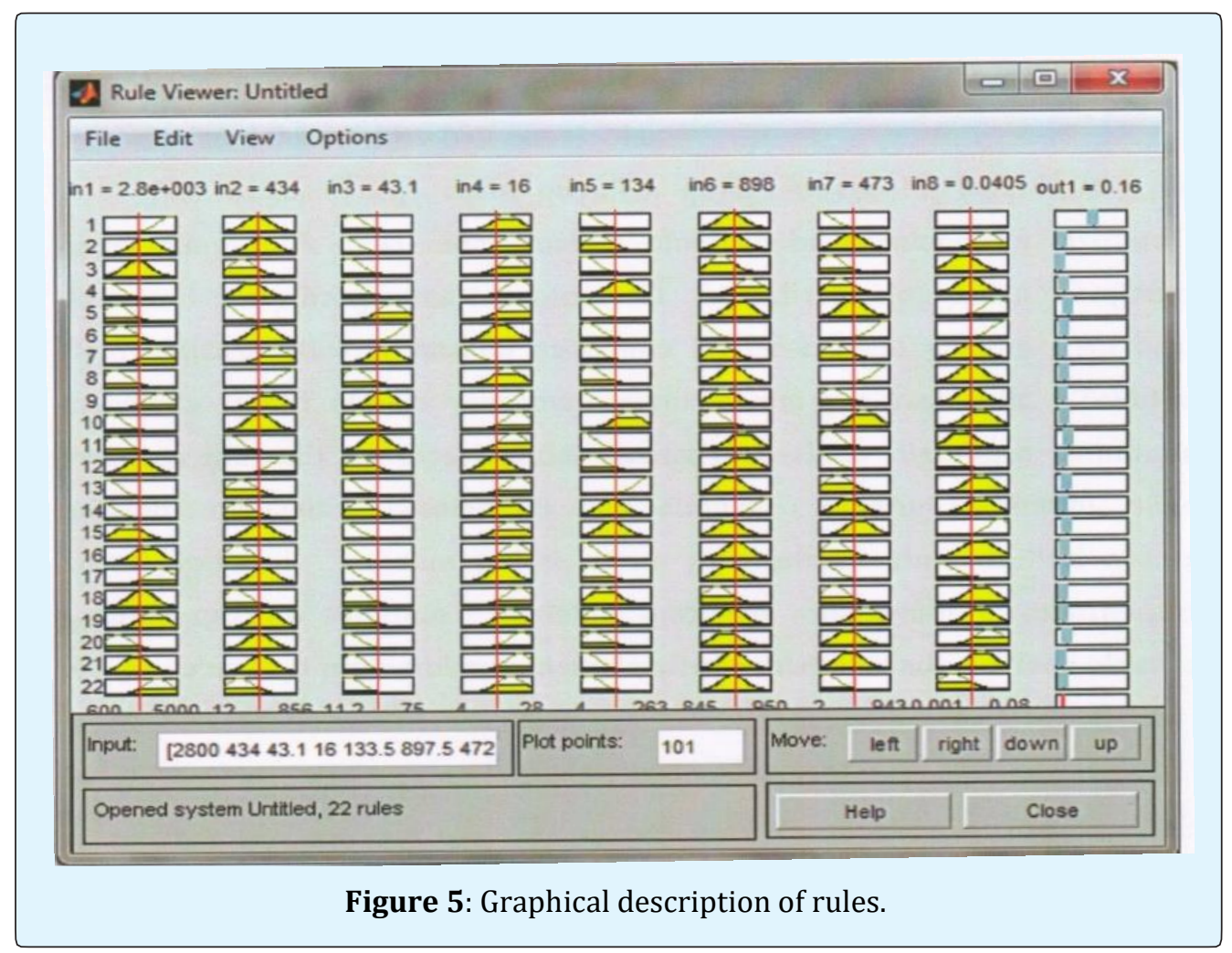

Expert can get information about oil production entering possible factors into input menu line described in Figure 5.

Soft computing and data mining technology used for intellectual analysis of data is favourable means for the specialists working in oil extraction field from the information processing and more precise decision making points of view.

Applying existing fuzzy clustering methods the solution of this problem makes it possible to determine precisely the number of the clusters. Let's mention that these methods being the main methods of data mining technology serve to get knowledge from data and compiling "If...then..." rules $[7,8]$. It is possible to solve oil spheres development problems from information processing view point using fuzzy dimensions making more adequate description of the indefiniteness possible in clusters based "If...then..." type rules $[8,9]$.

If to consider that in indefiniteness condition as the drilling of deep wells undergoes to unknown indefinite initiating influences the drilling technology project should be distinguished from the real technology. In these cases, there is a great need in the effective control system. From this point, the approach offered in the paper is of great significance. The offered approach can be used in various types problems - to solve various engineering tasks for recognizing rocks in the drilling process, to set up the model of drilling rate considering factors as rocks classification, their features, bit types, regime parameters, also to design problems of controlling well drilling process.

\section{Conclusion}

The obtained results:

1. Statistic methods based analysis of complex technological process as drilling of wells is guided considering factors characterized by indefiniteness and the environment and the development of the control system of well drilling with the purpose of decision making in this condition.

2. The results obtained by the classic methods complex directed to the increasing of layer oil production coefficient in oil fields development have been precised using Fuzzy technology and Data mining technologies.

3. Application of fuzzy clusterization gives several possible variants and it widens choice of decision making alternatives. 


\section{Petroleum \& Petrochemical Engineering Journal}

\section{References}

1. Shirali I, Hasanov R (2019) Drilling of the wells innovative technics and technology. Lap-Lambert Academic publishing pp: 366.

2. Hasanov R, Hasanov A, Jamalov V (2008) Application of fuzzy logic methods for effective management of rehabilitation of breakdown wells. $6^{\text {th }}$ International Symposium on Intelligent \& Manufacturing systems, Turkey, pp: 66-70.

3. Siha S (1999) A classified model for applying the theory of constraints to service organizations. Managing service Quality 9(4): 255-264.

4. Kaky BK, Rachamadugu R (1992) Layout design for flexible manufacturing systems. European Journal of operational Research 57(2): 224-230.

5. Tae DB, So BS, Jung YH, Lee DW, Park DH (2008) Planning a linear trajectory in a singular area of fiveaxis milling by step-length optimization. $6^{\text {th }}$ International Symposium on Intelligent \&
Manufacturing systems, Sakarya, Turkey, pp: 317327.

6. Nagy G, Salhi S (2007) Location-routing: issues, models and methods. European Journal of Operational Research 177(2): 649-672.

7. Barbarosoglu G, Yazgac T (1997) An Application of the Analytic Hierarchy Process to the Supplier. Selection Problem, Production and Inventory Management Journal 38(1): 14-21.

8. Apte C, Welss S (1997) Data mining with decision trees and decision rules. Future Generation Computer Systems 13(2-3): 197-210.

9. Erensal YC, Esra Y (2008) An Integrated Decision Support Framework for System Performance Prediction and Improvement. $6^{\text {th }}$ International Symposium on Intelligent \& Manufacturing systems, Sakarya, Turkey, pp: 689-694. 